\title{
Inverse and Disjoint Connected Cototal Domination number of the Jump Graph of a Graph
}

\author{
Annie Jasmine S.E. ${ }^{1}$, K. Ameenal Bibi ${ }^{2}$ \\ ${ }^{1}$ Department of Mathematics, Voorhees College, Vellore - 632001. \\ Email: annie.jasmine.se@gmail.com. \\ ${ }^{2}$ Department of Mathematics, D.K.M College for women (Autonomous), \\ Vellore-632001.
}

\begin{abstract}
Let $D \subseteq V[J(G)]$ be a connected cototal dominating set of $J(G)$, if $\langle V[J(G)]-D\rangle \neq \emptyset$ contain a dominating set $D^{\prime}$ such that $\left.<V[J(G)]-D^{\prime}\right\rangle$ has no isolated vertex and $\left\langle D^{\prime}\right\rangle$ is connected, then $D^{\prime}$ is the inverse connected cototal dominating set of $J(G)$ with respect to $D$. The minimum cardinality of a minimal inverse connected cototal dominating set is termed as, the inverse connected cototal domination number, denoted by $\gamma_{c c t}[J(G)]$. Exact values of some standard graphs, bounds and the relationship of this parameter with other graph theoretic graph parameters are evaluated.

Keywords: Inverse Domination number of the jump graph of a graph, Inverse Connected Cototal Dominating set of a jump graph, Inverse Connected Cototal Domination number of a jump graph, Disjoint Connected Cototal Domination number of a jump graph.
\end{abstract}

\section{Mathematics Subject classification: 05 C69.}

\section{INTRODUCTION:}

All graphs G(p,q) considered here are simple,finite, connected, undirected with order $\mathrm{p}$ and size $\mathrm{q}$. For all other notations and terminologies we refer [1].

A graph whose vertex set is the edge set of a graph $G$ is called as a line graph $\mathbf{L}(\mathbf{G})$. Two vertices are adjacent in $L(G)$ if and only if the corresponding edges are adjacent in $\mathrm{G}$. The graph defined on the edge set $E(G)$ where two vertices are adjacent if and only if the corresponding edges in $\mathrm{G}$ are non-adjacent is referred as Jump graph $\boldsymbol{J}(\boldsymbol{G})$ of the graph G. Thus, jump graph is the complement of line graph. Hence the isolated vertices of $\mathrm{G}$, if it exist, has no part in $J(G)$.

A non-empty subset $D$ of the vertex set $\mathrm{V}[J(G)]$ is a dominating set of $J(G)$, if every vertex not in $D$ is adjacent to atleast one vertex in $D$. The minimum cardinality of a minimal dominating set of $J(G)$ is the dominating set of $J(G)$ and the cardinality is the domination number of $J(G)$, denoted by $\gamma[J(G)]$. Imposing restrictions on the dominating set $D$, various domination parameters have been defined. When $\langle D\rangle$ is connected then $D$ is a connected dominating set of $J(G)$ and the minimum cardinality of a minimal connected dominating set is the connected domination number, $\gamma_{c}[J(G)]$.

Restriction on the complement set $\{V[J(G)]-D\}$ of the jump graph define many parameters. If there exist a dominating set $D^{\prime}$ in $\{V[J(G)]-D\}$ then $D^{\prime}$ is the inverse dominating set with respect to the dominating set $D$. When the induced subgraph of the inverse dominating set $D^{\prime}$ is connected, then $D^{\prime}$ is the inverse connected dominating set of $\mathrm{G}$, denoted by $\gamma_{c}^{-1}(G)$.

A dominating set $D$ is a cototal dominating set of $J(G)$ if $\langle V[J(G)]-D\rangle \neq \emptyset$ contains no isolated vertex. The minimal cototal dominating set with minimum cardinality is the cototal domination number of $J(G)$, denoted as $\gamma_{c t}[J(G)]$.

The disjoint domination number, $\gamma \gamma(G)$ of $\mathrm{G}$ is the minimum cardinality of two disjoint dominating sets in $\mathrm{G}$ [9].

In this paper, we discussed about the inverse connected cototal domination number of a jump graph and the disjoint connected cototal domination number of a jump graph have been carried.

Here, we have considered, simple connected graph G of size $|E|=q \geq 4$.

\section{Inverse Connected Cototal Domination number of the Jump Graph of a Graph:}

Definition: 2.1.

Let $D \subseteq V[J(G)]$ be a connected cototal dominating set of the jump graph of G. In $[V[J(G)]-D]$, if there exists a dominating set $D^{\prime}$ such that $<V[J(G)]-D^{\prime}>$ contains no isolated vertex and $\left\langle D^{\prime}\right\rangle$ is connected, then $D^{\prime}$ is the inverse connected cototal dominating set of $J(G)$ with respect to $D$. The minimum cardinality of $D^{\prime}$ is the inverse connected cototal domination number, denoted by $\gamma_{c c t}^{-1}[J(G)]$. 


\section{Available online at www.ijrat.org}

\section{Definition: 2.2}

If the cardinality of $D^{\prime}$ is maximum with respect to the minimality condition, then $D^{\prime}$ is known as the upper inverse connected cototal dominating set. Thus, $\left|D^{\prime}\right|=\Gamma_{c c t}[J(G)]$ is called the upper inverse connected cototal domination number.

\section{Theorem: 2.3.}

Exact values of Some Standard graphs.

$$
\begin{aligned}
& \text { (i). For } p \geq 6 \text {, } \\
& \text { (ii).For } p \geq 6 \text {, } \\
& \gamma_{c c t}^{-1}\left[J\left(P_{p}\right)\right]=2 \\
& \text { (iii).For } p \geq 6 \text {, } \\
& \gamma_{c c t}^{-1}\left[J\left(C_{p}\right)\right]=2 \\
& \text { (iv). For } p=p_{1}+p_{2} \text {, } \\
& \gamma_{c c t}^{-1}\left[J\left(K_{p}\right)\right]=3 \\
& \text { (v). For } p \geq 6 \text {, } \\
& \text { (vi). For } p \geq 4 \text {, } \\
& \text { (vii). For } p \geq 4 \text {, } \\
& \gamma_{c c t}^{-1}\left[J\left(K_{p_{1}, p_{2}}\right)\right]= \begin{cases}3, & p_{1}=2, p_{2} \geq 4 \\
2, & p_{1}, p_{2} \geq 3\end{cases} \\
& \gamma_{c c t}^{-1}\left[J\left(W_{p}\right)\right]= \begin{cases}3, & p=6 \\
2, & p>6\end{cases} \\
& \gamma_{c c t}^{-1}\left[J\left(P_{p} \circ K_{1}\right)\right]=2 \text {, } \\
& \gamma_{c c t}^{-1}\left[J\left(C_{p} \circ K_{1}\right)\right]=2 \\
& \text { (ix). For a Fan graph , } F_{p}=P_{P-1}+K_{1}, \quad \gamma_{c c t}^{-1}[J(G)]= \begin{cases}3, & p=5 \\
2, & p>5\end{cases} \\
& \text { (x). For a Friendship graph } F_{p}, p \geq 3, \quad \gamma_{c c t}^{-1}[J(G)]=2 \\
& \text { (xi). For Petersen graph, } \mathrm{G}=(10,15), \quad \gamma_{c c t}^{-1}[J(G)]=2 \text {. }
\end{aligned}
$$

\section{Bounds of $\gamma_{c c t}^{-1}[J(G)]$ :}

Theorem: 3.1.

The connected cototal domination number of the jump graph $J(G)$ of $\mathrm{G}$ is

Theorem: 3.2.

$$
\gamma_{c c t}^{-1}[J(G)] \geq 2
$$

If the inverse connected cototal dominating set exist for the jump graph $J(G)$ of $\mathrm{G}$, then, $\quad \gamma_{c c t}^{-1}[J(G)] \geq$

\section{Proof:}

Let $D$ be the connected cototal dominating set of the jump graph of G, then $\quad|D|=\gamma_{c c t}[J(G)] \geq$ 2. If there exist a connected cototal dominating set $D^{\prime}$ in $\quad[V[J(G)]-D]$ then, $\left|D^{\prime}\right| \geq|D|$. Thus, $\gamma_{c c t}^{-1}[J(G)] \geq 2$.

Theorem:3.3.

Let $\mathrm{G}(\mathrm{p}, \mathrm{q})$ be any connected graph, then the inverse connected cototal dominating set exist for the jump graph $J(G)$ only if $q \geq 6$.

Proof:

Let $D$ be the connected cototal dominating set of $J(G)$, then by theorem 3.1, $\quad|D|=\gamma_{c c t}[J(G)] \geq$ 2 and $[V[J(G)]-D]$ is a non-empty without isolated vertex. If $[V[J(G)]-D]$, contain a connected cototal dominating set $D^{\prime}$, then $D^{\prime}$ is called the inverse connected cototal dominating set of $J(G)$ and by theorem 3.2, $\left|D^{\prime}\right|=\gamma_{c c t}^{-1}[J(G)] \geq 2$. Thus, the existence of inverse connected cototal dominating set $D^{\prime}$ implies that $\left[V[J(G)]-D^{\prime}\right]$ is a non-empty set containing no isolates. This means $\left|V[J(G)]-D^{\prime}\right| \geq 2$.

Theorem :3.4.

The inverse connected cototal dominating set does not exist for the jump graph of a connected graph $\mathrm{G}$, if $\mathrm{G}$ contains an edge with $\operatorname{deg}\left(e_{i}\right)=q-2, \mathrm{i}=1$ to $\mathrm{q}$.

\section{Proof:}

Let there exist an edge $e_{i}$ in the simple connected $\mathrm{G}$, such that $\operatorname{deg}\left(e_{i}\right)=q-2$. Then in the jump graph the vertex $v_{i}{ }^{\prime}$ corresponding to $e_{i}$ will be a pendent vertex. But every pendent vertex is a member of the connected cototal dominating set along with its support vertex. Thus there exists no inverse connected dominating set in $J(G)$. 


\section{Available online at www.ijrat.org}

\section{Observation:3.5.}

The inverse connected cototal dominating set does not exist for all the jump graphs of G.

\section{Theorem:3.6.}

If the inverse connected cototal dominating set exist, then,

Proof:

$$
\gamma_{c c t}[J(G)] \leq \gamma_{c c t}^{-1}[J(G)]
$$
dominating set.

Let $D^{\prime}$ be the inverse connected cototal dominating set of $J(G)$ then $D^{\prime}$ is also the connected cototal Theorem:3.7.

Let $J(G)$ be the jump graph of $\mathrm{G}$ with the inverse connected cototal dominating set, then,

$$
\gamma_{c c t}[J(G)]+\gamma_{c c t}^{-1}[J(G)] \leq q . \quad \text { Bound is sharp for } W_{5} .
$$

Proof:

We have considered $\mathrm{G}$ to be a simple connected graph, hence, $|E(G)|=q$. Then in $J(G),|V[J(G)]|=$ $|E(G)|=q$. By Ore[1], the theorem follows.

For the Wheel graph on 5 vertices, equality holds.

Parameters:

Relation between Inverse connected cototal domination of $J(G)$ with other graph theoretic Theorem: 3.8.

Let $\mathrm{G}$ be a connected graph and $J(G)$ be its jump graph, then $\gamma^{-1}[J(G)] \leq \gamma_{c t}^{-1}[J(G)] \leq \gamma_{c c t}^{-1}[J(G)]$. Bound is sharp for $P_{p}, C_{p}$.

Proof:

Every inverse connected cototal dominating set of $J(G)$ is the inverse cototal dominating set of $J(G)$ and also it is the inverse dominating set of $J(G)$.

Theorem:3.9.

For the jump graph of a connected graph G, $\gamma_{c}^{-1}[J(G)] \leq \gamma_{c c t}^{-1}[J(G)]$.

Theorem:3.10. (Inverse Domination Chain )

Theorem:3.11.

Let $\mathrm{G}$ be a connected graph and $J(G)$ be its jump graph, then

$\gamma^{-1}[J(G)] \leq \gamma_{c}^{-1}[J(G)] \leq \gamma_{c t}^{-1}[J(G)] \leq \gamma_{c c t}^{-1}[J(G)]$.

Let $\beta_{1}(G)$ denote the edge independence number of a connected graph $\mathrm{G}$, then

$$
\gamma_{c c t}^{-1}[J(G)] \leq \beta_{1}(G)
$$

\section{Proof:}

Let $\mathrm{E}=\left(e_{1}, e_{2}, \ldots . . e_{q}\right)$ be the edge set of $\mathrm{G}(\mathrm{p}, \mathrm{q}), \mathrm{q} \geq 6$. Let $D$ be the connected cototal domination number of $J(G)$ and let $\mathrm{S}=\left(e_{1}, e_{2}, \ldots . e_{n}\right)$ denote the maximum edge independent set of $\mathrm{G}$ with respect to $D$. Then in $J(G)$ the vertex set of the corresponding edges of the set $\mathrm{S}$ form a connected induced sub graph which is also a dominating set of $J(G)$. Hence, by the choice of $\mathrm{q}$ and $\mathrm{S}$, it is apparent that $\left\{V[J(G)]-D^{\prime}\right\}$ is non-empty and connected.

Theorem:3.12.[4]

For the jump graph $\mathrm{J}(\mathrm{G})$ with inverse connected cototal dominating set , $\quad \gamma_{c c t}^{-1}[J(G)] \leq q-\Delta^{\prime}(G)$. Equality holds for $G \cong K_{2, p}, p \geq 4$.

\section{Theorem: 3.13[ 5]}

\section{Proof:}

If $G \cong T$, and $\operatorname{diam}(\mathrm{T})$ not less than 4 , then, $\gamma_{c c t}^{-1}[J(G)]=2$

Consider a tree $\mathrm{T}$ having diameter greater than 3, otherwise, the jump graph of $\mathrm{T}$ will have isolated vertices. Let $D$ be the connected cototal dominating set. Thus in $\mathrm{T}$, with respect to connected cototal dominating set there exists vertices $v_{j}$ and $v_{k}$ with maximum distance between them. In $J(T)$, the vertices corresponding to the edges $e_{j}$ and $e_{k}$ adjacent to $v_{j}$ and $v_{k}$ form the minimum connected cototal dominating set of $J(T)$ with respect to connected cototal dominating set.

\section{Disjoint Connected Cototal Domination number of the Jump Graph of a graph:} Definition:4.1.[4] 


\section{Available online at $w w w . i j r a t . o r g$}

Let $D_{1}$ and $D_{2}$ be two disjoint connected cototal dominating sets of $J(G)$ of $\mathrm{G}$. Then the minimum cardinality of the union of two disjoint minimal connected cototal dominating set of $J(G)$ is called the disjoint connected cototal domination number, denoted by, $\gamma_{c c t} \gamma_{c c t}[J(G)]$.

(i.e) $\gamma_{c c t} \gamma_{c c t}[J(G)]=\operatorname{mini}\left\{\left|D_{1}\right|+\left|D_{2}\right|\right\}$.

The two disjoint connected cototal dominating set whose union has the cardinality $\gamma_{c c t} \gamma_{c c t}[J(G)]$ is called $\gamma_{c c t} \gamma_{c c t}[J(G)]-$ pair.

\section{Theorem:4.2.}

Exact values of $\boldsymbol{\gamma}_{c c t} \gamma_{c c t}[J(G)]$ - for some standard graphs:

$$
\begin{aligned}
& \text { 1. } \gamma_{c c t} \gamma_{c c t}\left[J\left(P_{p}\right)\right]=4, \quad p \geq 6 \\
& \text { 2. } \gamma_{c c t} \gamma_{c c t}\left[J\left(C_{p}\right)\right]=4, \quad p \geq 6 \\
& \text { 3. } \gamma_{c c t} \gamma_{c c t}\left[J\left(K_{p}\right)\right]=6, \quad p \geq 6 \\
& \text { 4. } \gamma_{c c t} \gamma_{c c t}[J(G)]=\left\{\begin{array}{cc}
8, & \text { for } G \cong K_{m, n}, \quad m=2, n \geq 4 \\
6, & \text { for } G \cong K_{m, n}, \quad m, n \geq 3
\end{array}\right. \\
& \text { 5. } \gamma_{c c t} \gamma_{c c t}\left[J\left(W_{p}\right)\right]=\left\{\begin{array}{cc}
8, & p=5 \\
6, & p=6 \\
4 & p \geq 7
\end{array}\right. \\
& \text { 6. } \gamma_{c c t} \gamma_{c c t}[J(G)]=4, G \cong P_{p} \circ K_{1} \cdot p \geq 4 \\
& \text { 7. } \gamma_{c c t} \gamma_{c c t}[J(G)]=4, G \cong C_{p} \circ K_{1} \\
& \text { 8. For petersen graph, } \gamma_{c c t} \gamma_{c c t}[J(G)]=4 .
\end{aligned}
$$

Theorem:4.3.

\section{Proof:}

Let $J(G)$ be the jump graph of a graph $\mathrm{G}$, then

$\gamma_{c c t} \gamma_{c c t}[J(G)] \leq q$. Equality holds for $K_{2,4}$ and $W_{5}$. the theorem follows.

\section{Theorem: 4.4 .}

Exact value(Theorem 4.2) proves the equality.

Let $\mathrm{G}$ be a connected graph, then, every disjoint connected cototal dominating set of $J(G)$ is the disjoint total dominating set of $J(G)$.

(i.e) $\gamma_{c c t} \gamma_{c c t}[J(G)]=\gamma_{t} \gamma_{t}[J(G)]$, where $\gamma_{t}$ is the total dominating set of $\mathrm{G}$.

Proof:

Let $D_{1}$ and $D_{2}$ be two disjoint dominating set of $\mathrm{J}(\mathrm{G})$, with $\left\langle D_{1}\right\rangle$ and $\left\langle D_{2}\right\rangle$ are connected. If $<$ $V[J(G)]-D_{1}>$ and $\left\langle V[J(G)]-D_{2}>\right.$ contain no isolated vertices then $D_{1}$ and $D_{2}$ are the connected cototal dominating set of $J(G)$. Thus the two disjoint dominating set $D_{1}$ and $D_{2}$ are the total dominating set of $\mathrm{J}(\mathrm{G})$.

Theorem: $\mathbf{4 . 5}$.

For any connected graph $\mathrm{G}, \quad 2 \gamma_{c c t}[J(G)] \leq \gamma_{c c t} \gamma_{c c t}[J(G)]$.

\section{Proof:}

Equality holds for the standard graphs given in Theorem 4.2.

For a connected graph $\mathrm{G}$, if its jump graph contains two disjoint connected cototal dominating sets $D_{1}$ and $D_{2}$ then, $\left|D_{1}\right| \leq\left|D_{2}\right|$, since both the sets are minimum cardinality sets.

\section{Theorem: 4.6.}

Equality holds for the graphs given in theorem 4.2

For the jump graph $J(G)$ of the graph $\mathrm{G}$, with inverse connected cototal dominating set,

Theorem: 4.7.[4]

$$
2 \gamma_{c c t}[J(G)] \leq \gamma_{c c t}[J(G)]+\gamma_{c c t}^{-1}[J(G)]
$$

Equality holds for $P_{p}, C_{p}, K_{p}(p \geq 6)$.

For the connected graph $\mathrm{G}(\mathrm{p}, \mathrm{q}), \quad \gamma \gamma[J(G)] \leq \gamma_{c c t} \gamma_{c c t}[J(G)]$.

\section{Theorem: 4.8 .}

$$
\text { Bounds are sharp for } P_{p}, C_{p} \text { and } K_{p}(p \geq 6) \text {. }
$$

Let $J(G)$ be the jump graph of $\mathrm{G}$, then, $\gamma_{c t} \gamma_{c t}[J(G)] \leq \gamma_{c c t} \gamma_{c c t}[J(G)]$. 


\section{Available online at www.ijrat.org}

\section{Theorem: 4.9.}

\section{Proof:}

Let $G \cong T$, then for $J(T)$ with $\operatorname{diam}(T)>3$, we have

$$
\gamma \gamma[J(T)]=\gamma_{c t} \gamma_{c t}[J(T)]=\gamma_{c c t} \gamma_{c c t}[J(T)] \text {. }
$$

For a tree graph $\mathrm{T}$, let $J(T)$ has $\gamma_{c c t}^{-1}[J(T)]$-set. This implies we have two disjoint connected cototal dominating set in $J(T)$. Moreover, for a tree T with $\operatorname{diam}(T)>3$, there exists two disjoint pair of vertices in $J(T)$ corresponding to two disjoint pair of edges $\left(e_{1}, e_{q-1}\right)$ and $\left(e_{2}, e_{q}\right)$ which form the minimal dominating set of $J(T)$. Also $<V[J(T)]-D_{1}>$ and $\quad<V[J(T)]-D_{2}>$ has no isolated vertices. Thus $D_{1}$ and $D_{2}$ are the two disjoint cototal dominating set of $J(T)$. By the choice of T, $\left\langle D_{1}\right\rangle$ and $\left\langle D_{2}\right\rangle$ is connected. Thus $D_{1}$ and $D_{2}$ are the disjoint connected cototal dominating set.

\section{Definition: 4.10.[4]}

The jump graph $J(G)$ of a connected graph $\mathrm{G}$ is $\gamma_{c c t} \gamma_{c c t}-$ minimum if $\quad \gamma_{c c t} \gamma_{c c t}[J(G)]=2 \gamma_{c c t}[J(G)]$.

Definition:4.11.[4]

The jump graph $J(G)$ of a connected graph $\mathrm{G}$ is $\gamma_{c c t} \gamma_{c c t}[J(G)]$-maximum if $\gamma_{c c t} \gamma_{c c t}[J(G)]=q$.

\section{Definition:4.12.[4]}

The jump graph $J(G)$ of a connected graph $\mathrm{G}$ is $\gamma_{c c t} \gamma_{c c t}[J(G)]-$ strong if $\gamma_{c c t} \gamma_{c c t}[J(G)]=2 \gamma_{c c t}[J(G)]=q$.

Example:4.13.

(i). For $\mathrm{p} \geq 6$, all the standard graphs given in theorem. 4.2, are $\gamma_{c c t} \gamma_{c c t}-$ minimum.

(ii). The graphs, $K_{2,4}$ and $W_{5}$ are $\gamma_{c c t} \gamma_{c c t}[J(G)]$-maximum

(iii). When $\mathrm{G}$ is either $K_{2,4}$ or $W_{5}$, then $\mathrm{G}$ is $\gamma_{c c t} \gamma_{c c t}[J(G)]$ - strong.

\section{REFERENCES}

[1]. T.H.Haynes, S.T.H Hedetniemi and P.J. Slater, Fundamentals of domination in graphs, Marcel Dekker Inc., New York (1998).

[2]. V.R.Kulli, Theory of domination in graphs, Vishwa International publications, Gulbarga, India (2010 ).

[3].V.R.Kulli, B.Jaakiram and Radha R.Iyer, Cototal Domination number of a graph, Journal of Discrete Mathematical Sciences \& Cryptography. Vol2(1992)pp.179-184.

[4].V.RKulli and S.C.Sigarkanti, Inverse domination in Graph, Nat. Acad. Sci. Lett., 14 (1991), 473-475.

[5]. Y.B.Maralabhavi, Anupama S.B.,Venganagouda M.Goudar, Domination number of Jump graph, International Mathematical Forum, Vol 8,No.16, 753-758(2013).

[6].M.Karthikeyan, A.Elumalai, Inverse Domination number of a Jump graph, International journal of Pure and Applied Mathematics, Vol 103, No.3 (2015),477-483.

[7].Anupama S.B., Y.B.Maralabhavi, Venkanagouda M.Goudar, Connected Domination Number of a Jump Graph, Journal of Computer and Mathematical Sciences, Vol 610 (2015), 538-545.

[8]. Annie Jasmine S.E., K.Ameenal Bibi., Inverse connected and disjoint connected domination number of a jump Graph , international Journal of Engineering and Tecnology (UAE).

[9]. V.R.Kulli and S.C.Sigarkanthi, Inverse domination number in graphs, Nat. Acad.Sci.Lett.,14(1991) 473-475.

[10]. G.S.Domke, J.EDunbar and L.R.Markus, Inverse domination number of a graph, Ars Combin.,72(2004) 149160. 